\title{
Meta-análisis: Las estatinas reducen el riesgo de enfermedad coronaria y mortalidad total independientemente del sexo y la edad
}

Effect of statins on risk of coronary disease.

LaRosa J, He J, Vupputuri S. JAMA 1999; 282:2340-46.

\section{Objetivo}

Estimar la reducción de riesgo de enfermedad coronaria y mortalidad total asociada al tratamiento con estatinas, particularmente en ancianos y mujeres.

\section{Fuente de Datos y Selección de Estudios}

Se realizó una búsqueda MEDLINE, en revistas de idioma Inglés, desde 1966 a diciembre de 1998 (además de referencias bibliográficas y de autores) de estudios clínicos randomizados a tratamiento con estatinas o placebo durante al menos 4 años. Los puntos finales primarios de dichos estudios para su inclusión en este meta-análisis debían ser: eventos coronarios mayores o muerte coronaria.

\section{Extracción de datos}

Dos de los autores consignaban en forma independiente la información sobre tamaño muestral, duración, tipo y dosis de tratamiento hipolipemiante, características basales de los pacientes, reducción lipídica durante la intervención y resultados siguiendo un protocolo estandarizado y resolviendo las discordancias por consenso.

\section{Resultados principales}

Cinco estudios con un total de 30.817 participantes reunían los criterios de inclusión (2 de prevención primaria con 13.200 pacientes y 3 de prevención secundaria con 17.617 pacientes con historia de enfermedad coronaria). La media de seguimiento fue 5,4 años con una media de edad de 59 años. El tratamiento con estatinas se asoció a una reducción de $20 \%$ del colesterol total, de $28 \%$ en LDL-C, de $13 \%$ en triglicéridos y un aumento de $5 \%$ en HDL-C.

La reducción de eventos el período de seguimiento puede verse en la tabla. Comparando con el grupo control, el tratamiento activo se asoció a una reducción del riesgo de enfermedad coronaria evitando 36 eventos y 13 muertes por cada mil pacientes tratados.

\begin{tabular}{|c|c|c|c|c|c|c|}
\hline Resultados & Placebo & Estatinas & $\begin{array}{l}\text { RRR \% } \\
\text { (IC95\%) }\end{array}$ & $\begin{array}{l}\text { RRA \% pac. } \\
(\text { IC95\%) }\end{array}$ & $\begin{array}{c}\text { NNT } \\
\text { (IC95\%) }\end{array}$ & $\mathrm{P}$ \\
\hline $\begin{array}{l}\text { Eventos } \\
\text { coronarios }\end{array}$ & 2042 & 1490 & $31(26-36)$ & $36(29-31)$ & $28(23-34)$ & $<0,001$ \\
\hline $\begin{array}{l}\text { Muertes } \\
\text { coronarias }\end{array}$ & & & $29(20-36)$ & $13(9-18)$ & $75(56-112)$ & $<0,001$ \\
\hline $\begin{array}{l}\text { Muertes } \\
\text { cardiovas- } \\
\text { culares } \\
\end{array}$ & 868 & 646 & $27(1$ & $14(10$ & $69(52-103)$ & $<0,001$ \\
\hline $\begin{array}{l}\text { Muertes } \\
\text { No cardio- } \\
\text { vasculares }\end{array}$ & - & & $7(-7$ a 19$)$ & $2(-2$ & & $<0,001$ \\
\hline $\begin{array}{l}\text { Muertes } \\
\text { Totales }\end{array}$ & 1297 & 1046 & $21(14-28)$ & $16(11-22)$ & $1(56-112)$ & 01 \\
\hline
\end{tabular}

*ver glosario para RRR, RRA y NNT.

Los estudios de prevención primaria mostraron una RRR de eventos coronarios mayores de 34\% (IC95\% 23-43\%, $\mathrm{p}<0.001$ ), una reducción de la mortalidad cardiovascular (0R 0,68 IC95\%, 0,50-0,93; p=0.01) y total, pero no significativa en el último caso (0R 0,87 IC $95 \%, 0,71-1,06 ; p=0.18)$. Los estudios de prevención secundaria mostraron una RRR de eventos coronarios mayores de 30\% (IC95\%, 24-35\% p 0.001), una menor mortalidad cardiovascular (OR 0,73 IC95\%, 0,66-0,82; $\mathrm{p}<0.001)$ y total (OR 0,77 IC95\%, 0,70-0,85; $\mathrm{p}<0.001)$. Las estatinas, tanto en prevención primaria como secundaria, no se asociaron a modificaciones en la mortalidad no cardiovascular.

El análisis de subgrupos mostró que la reducción de riesgo fue similar para mujeres y hombres, 29\% (IC95\% 13-42\% p<0.001) y 31\% (IC95\% 26$35 \% \mathrm{p}<0.001$ ) respectivamente.

La reducción de riesgo absoluto también fue estadísticamente significativa en 4 de los 5 estudios. La reducción de riesgo relativo fue similar para mayores y menores de 65 años, 32\% (IC95\% 23-39 p<0.001) y 31\% (IC95\% 24-33\% $p<0.001$ ) respectivamente.

\section{Conclusiones}

Este meta-análisis indica que la reducción del LDL-C asociado al tratamiento con estatinas, disminuye el riesgo de enfermedad coronaria y mortalidad total. La reducción de riesgo fue similar para hombres y mujeres y para individuos mayores y menores de 65 años.

\section{COMENTARIO}

Los resultados de los trabajos realizados en las diferentes etapas de la prevención cardiovascular justifican cada vez más un manejo agresivo de los niveles de colesterol en pacientes con o sin enfermedad coronaria. Los pacientes en prevención secundaria con alteraciones en el perfil lipídico, tienen un riesgo de entre 5 y 7 veces mayor de desarrollar un nuevo evento cardiovascular, y sólo un tercio de ellos recibe terapia hipolipemiante, según datos no publicados del National Heart, Lung, and Blood Institute. Datos similares de subtratamiento se observaron también en la prevención primaria, especialmente en el subgrupo de pacientes con alto riesgo cardiovascular. Esto lleva a que un importante número de pacientes son privados de recibir un tratamiento costo-efectivo para la reducción de riesgo de un nuevo evento. En el caso de la prevención secundaria, para lograr una significativa disminución de la morbili- dad y de mortalidad en la enfermedad aterosclerótica, el manejo agresivo de los niveles de colesterol debe iniciarse cuando se establece el diagnóstico clínico de enfermedad aterosclerótica ${ }^{1}$. Esto está claramente documentado en los estudios $4 S$ y CARE, los cuales muestran una reducción de riesgo a los 2 años de iniciada la terapia hipolipemiante. Las evidencias muestran que los beneficios de esta terapéutica son independientes del sexo y de la edad.

Las estatinas, a su vez, han demostrado tener propiedades sobre la función endotelial, componentes celulares de la placa aterosclerótica, la trombosis y la inflamación. Por lo tanto las posibilidades de que en un futuro las estatinas sean consideradas algo más que simples agentes reductores de colesterol e incluidas entre las principales drogas para el tratamiento de la enfermedad coronaria, no se encuentran lejanas.

\section{Dra. Nora E. Vainstein}

Centro de Prevención de Enfermedades Cardiovasculares. Servicio de Cardiología. Hospital Italiano de Buenos Aires 